\title{
11 Anhang zur Orientierung: Die wichtigsten Operationen der südosteuropäischen Waffen-SS Verbände
}

Nicht nur die ethnisch-religiöse und politische Vielfalt verschiedener Widerstandsgruppen, sondern auch die topographische Ausgangslage stellten die Verantwortlichen der Waffen-SS, Phleps, Sauberzweig oder Schmidhuber, hinsichtlich der Einsätze vor Schwierigkeiten: Auf dem Balkan finden sich mediterrane Küstengebiete, die sich entweder flach oder aber oft klippenartig gestalten. Zahlreiche Gebiete Kroatiens, Bosniens, Montenegros, Serbiens, Kosovos und Albaniens sind von Karstgebirgen geprägt, die sich steil und schroff abfallend, aber auch sanft hügelig in den Hochebenen gestalten können. Weitere Teile bestehen aus Ebenen, etwa große Flächen Serbiens. Inmitten dieser Extreme liegen weitgezogene Hügellandschaften mit dichten Wäldern. Ein kontinentales Klima im Innern Südosteuropas wechselt sich ab mit maritimen Klimazonen an den Küsten und dem küstennahen Binnenland. ${ }^{1}$

Politisch gesehen entsprach der Balkan einem Puzzle, das sich aus sehr unterschiedlichen politischen Staatsgebilden zusammensetzte: Staaten wie der NDH, Rumänien, Bulgarien oder Ungarn zählten zu den Verbündeten der Nationalsozialisten und konstituierten sich als Diktaturen bzw. Marionettenregime. „Großalbanien“ kam zwar im September 1943 unter deutsche Herrschaft, galt aber vordergründig als „neutraler Staat“, in welchem, so Neubacher, „die deutsche Wehrmacht [...] als Freund des albanischen Volkes Anspruch auf gastfreundliche Behandlung“ erhob. ${ }^{2}$ Serbien hingegen verblieb seit 1941 unter deutscher Militärherrschaft.

Einsätze gegen Partisanen machten jedoch vor neuen und alten Landesgrenzen nicht halt. Im Gegenteil, die Partisanen versuchten, topographisch schwierig kontrollierbare Grenzgebiete für eigene Zwecke zu nutzen - beispielsweise in den

1 Der OB Südost von Weichs schreibt von ,riesigen Frontbreiten“, welche durch die in Südosteuropa stationierten Divisionen abgedeckt werden mussten. OB Südost (Heeresgr. F) Generalfeldmarschall Maximilian von Weichs, H.Q., „Die große Absetzbewegung im Südosten“, Januar 1945, BArchF, RH 19-XI/86, S. 2. Zur topographischen Ausgangslage vgl. beispielsweise Schmider, Der jugoslawische Kriegsschauplatz, S. 1009f. Vgl. auch Calic, Geschichte Jugoslawiens, S. 137-170 und Peter Jordan (Hg.): Albanien. Geographie, historische Anthropologie, Geschichte, Kultur, postkommunistische Transformation.

2 Neubacher, Sonderauftrag Südost, S. 113. Vgl. Zaugg, Albanische Muslime, S. 93.

Anmerkung: Ausgenommen ist die „Kama“, die an diesen Operationen nicht teilnahm.

Ә Open Access. (c) 2021 Franziska Anna Zaugg, publiziert von De Gruyter. (cc)BY Dieses Werk ist lizenziert unter einer Creative Commons Namensnennung 4.0 International Lizenz. 
Grenzgebieten in und um Kosovo und Sandžak oder den Grenzgebieten Ostbosniens zu Serbien. ${ }^{3}$ In dieser komplexen Umgebung arbeiteten Wehrmacht und Waffen-SS trotz anhaltender Streitigkeiten über Personalkontingente in vielen Operationen zusammen. Eine Zusammenarbeit kann auch mit der 1. Gebirgs-Division nachgewiesen werden. ${ }^{4}$ Roland Kaltenegger schrieb in seinem Buch „Totenkopf und Edelweiß“ zur Zusammenarbeit zwischen den in Südosteuropa stationierten Verbänden: „Mit einem engen Schulterschluss zwischen den Gebirgsjägern der Wehrmacht und Waffen-SS hatte das Kriegsjahr 1943 geendet und so begann auch das neue Jahr 1944. Die unverbrüchliche Waffenkameradschaft im Zeichen des Edelweiß steigerte sich fortan von Einsatz zu Einsatz, da man wiederholt aufeinander angewiesen war und genau wusste, dass man sich in jeder Lage aufeinander verlassen konnte. ${ }^{* 5}$ Die südosteuropäischen Waffen-SS-Verbände waren in zahlreichen Unternehmen mit klingenden Namen wie „Wegweiser“, „Osterei“, „Maibaum“, „Hackfleisch“, „Rösselsprung“ oder „Draufgänger“ eingesetzt - die einerseits die Vernichtung der kommunistischen Partisanen, oder wie beim Unternehmen „Schwarz“ der Četniks zum Ziel hatte. Die Erfolgsbilanz der Operationen kann insgesamt als mäßig bezeichnet werden, viele mussten frühzeitig abgebrochen werden und diejenigen im zweiten Halbjahr 1944 gerieten zum Desaster. Schmider hat in seinem Aufsatz „Auf Umwegen zum Vernichtungskrieg?“ eindrücklich die Wellenbewegungen der Gewalt nachgezeichnet, die sich während dieser vier Jahre in den verschiedenen Unternehmen ereigneten: Die Entwicklung verlief ausgehend von einem radikalen Vorgehen gegenüber Partisanen und Zivilisten mit Sühnekontingenten von 1:100 und 1:50, zu gegenseitigen Gefangenaustauschen zurück zur Erschießung von Gefangenen und schließlich auch der Straffreiheit von Überläufern. ${ }^{6}$ Die Gewaltexzesse während einiger dieser Operationen waren enorm und wurden nur durch den Vernichtungskrieg im Osten übertroffen. ${ }^{7}$ Die wichtigsten Operationen sollen im Folgenden kurz beschrieben und auf die Zusammenarbeit der südosteuropäischen Divisionen bei einigen dieser Unternehmen hingewiesen werden.

3 Vgl. Schmider, Auf Umwegen zum Vernichtungskrieg?, 905f. Vgl. Zaugg, Albanische Muslime, S. 255.

4 Vgl. Meyer, Blutiges Edelweiß, S. 557-560, 656, 667.

5 Kaltenegger, Totenkopf und Edelweiss, S. $181 \mathrm{f}$.

6 Vgl. Schmider, Auf Umwegen zum Vernichtungskrieg?, S. 900, $903 \mathrm{f} ., 907 \mathrm{f} ., 912 \mathrm{f}$.

7 Vgl. ebd. S. 919. 


\subsection{Operation „Weiß““ (I, II, III), 20. Januar bis März 1943}

Am 20. Januar 1943 begann die Operation „Weiß I“, auch Schlacht an der Neretva genannt, welche sich auf Bosnien-Herzegowina konzentrierte und bis Mitte März dauerte. ${ }^{8}$ In dieser Operation, welche die Eliminierung der Partisanen im Raum Westbosnien zum Ziel hatte, nahmen neben der Division „Prinz Eugen“ auch die 369. Infanterie-Division, die 717. Infanterie-Division und die 714. Infanterie-Division teil. Daneben waren drei italienische Divisionen sowie kroatische und als Hilfstruppen Četnik-Einheiten im Einsatz. ${ }^{9}$ Am 27. Januar 1943 gelang es der „Prinz Eugen“, Bihać zu besetzen. Danach marschierte sie zuerst Richtung Bohac - Bosanski Petrovac und von da nach Drvar mit dem Ziel Bosanski Grahovo. Am 25. Februar 1943 begann „Weiß II“. Drvar wurde aufgrund der schlechten Wetterverhältnisse erst am 27. Februar besetzt, Tito konnte die Stadt rechtzeitig verlassen. Ab März als „Weiß III“ bezeichnet, erfolgte am 3. März der Angriff auf Bosanski Grahovo, am 15. März erreichte die Division „Prinz Eugen“ Mostar. ${ }^{10}$ Das Kräfteverhältnis während dieser Operation fiel noch eindeutig zugunsten der Achsenmächte aus: Ungefähr 150.000 gegen schätzungsweise 20.000 Mann. $^{11}$

\subsection{Operation „Schwarz“, 15. Mai bis 16. Juni 1943}

Otto Kumm schreibt in seinem apologetischen Werk: „Endlich ist der Befehl des General-Kommandos Lüters da - am 15.5.1943 beginnt das Unternehmen ,Schwarz““.12 Das Unternehmen „Schwarz“ in Südost-Bosnien, auch Schlacht an der Sustjeska genannt, dauerte vom 15. Mai bis 16. Juni 1943. Neben der „Prinz Eugen“ waren auch die 1. Gebirgs-Division, die 104. Jäger-Division und die 369. Infanterie-Division eingesetzt. Bei dieser Operation sollten die wichtigsten Partisanenbasen in Montenegro zerstört werden. Sie galt in gleicher Weise sowohl den Partisanen als auch den Četnik-Verbänden. ${ }^{13}$ Der Angriff erfolgte von Mostar her entlang der Duga, da das Oberkommando der Wehr-

8 Vgl. Casagrande, Volksdeutsche SS-Division, S. 244.

9 Vgl. zum Umgang mit den Četniks als Hilfstruppen ebd. S. 245.

10 Vgl. Michaelis, Gebirgs-Divisionen der Waffen-SS, S. $69 \mathrm{f}$.

11 Vgl. ausführlich zu den Operationszyklen „Weiß I“, „Weiß II“ und „Weiß III“ Schmider, Partisanenkrieg, S. 206-261.

12 Kumm, Vorwärts Prinz Eugen, S. 73.

13 Vgl. Schmider, Auf Umwegen zum Vernichtungskrieg?, S. 911. 
macht annahm, dass eine Landung der Alliierten kurz bevorstehen würde. ${ }^{14}$ Auch in diesem Unternehmen waren die Kräfteverhältnisse noch klar: 127.000 Soldaten der deutschen, italienischen, kroatischen und bulgarischen Streitkräfte standen 16.000 Partisanen gegenüber. ${ }^{15}$ Phleps profitierte bei der Planung der Operation „Schwarz“ von seinen topografischen Kenntnissen. Denn bereits vor dem Ersten Weltkrieg hatte er in seiner Generalstabszeit in Sarajevo ein sehr ähnliches Szenario ausgearbeitet - und konnte es nun mit der Division „Prinz Eugen“ und den bereits erwähnten Verbänden umsetzen. ${ }^{16}$

Zwischen dem 10. und 12. Juni 1943 gelang den Partisanen jedoch ein Ausbruch Richtung Norden. ${ }^{17}$ Olovo, Srebrenica und Zvornik wurden von ihnen innerhalb von 20 Tagen zurückerobert. ${ }^{18}$ Obwohl Kasche in einem Schreiben an Ribbentrop davon ausging, dass Tito enorme Verluste von 10-12.000 Mann zu beklagen hatte, gingen die Partisanen als Sieger aus der Operation hervor. Somit war ein weiterer Versuch, die nun stark anwachsende Partisanenarmee zu zerschlagen, gescheitert. ${ }^{19}$ Um die eigene Niederlage in ein besseres Licht zu rücken, bezeichnete Lüters die Gegner als fanatische Kämpfer, die sehr gut organisiert, mit ausgesprochen guter Moral und unter hervorragender Führung kämpfen und das Territorium außerordentlich gut kennen würden. ${ }^{20}$

\subsection{Operationen „Herbstgewitter I“/,Herbstgewitter II“, 23. Oktober bis Dezember 1943}

Bei diesen Operationen sollte das Gebiet vor der kroatischen Küste um die Halbinsel Pelješac unter anderen durch Einheiten der „Prinz Eugen“ von Partisanen „gesäubert“ werden. Das verstärkte SS-Freiwilligen-Gebirgsjäger-Regiment 13 sollte die Inseln Brač, Hvar, Korčula und die Halbinsel Pelješac besetzen. Vor allem die Kämpfe auf Pelješac forderten auf beiden Seiten hohe Verluste. Mit der zweiten „Herbstgewitter“-Operation sollten die Partisanen auf der Insel Korčula eliminiert werden, welche „Herbstgewitter I“ überlebt hatten. ${ }^{21}$

14 Vgl. Redzić, Bosnia and Herzegovina, 39.

15 Vgl. ebd.

16 Vgl. Kumm, Vorwärts Prinz Eugen, S. 73.

17 Vgl. Redzić, Bosnia and Herzegovina, 39.

18 Vgl. ebd.

19 Vgl. ebd., $39 \mathrm{f}$.

20 Vgl. ebd., 40.

21 Vgl. Michaelis, Gebirgs-Divisionen der Waffen-SS, S. 79-84. 


\subsection{Operationen „Kugelblitz“, „Schneesturm“ und „Waldrausch“, Dezember 1943 - Januar 1944}

Die Operation „Kugelblitz“ sollte die für die Partisanen erschwerten Verhältnisse im Winter ausnutzen und hatte zum Ziel, diese an einem Einmarsch nach Serbien zu hindern und sie in der Folge zu liquidieren. Ihr Beginn wurde aufgrund schlechter Wetterverhältnisse auf den 2. Dezember 1943 angesetzt. Dabei trugen die beiden Divisionen „Prinz Eugen“ und die 1. Gebirgs- Division aufgrund ihrer Übung in unwegsamem Gelände die Hauptlast. Die „Prinz Eugen“ stieß aus der Herzegowina, die 1. Gebirgs-Division aus dem Sandžak-Gebiet vor. ${ }^{22}$ Auf diese Weise sollten die Partisanen in einem Kessel östlich von Sarajevo zusammengetrieben und umgebracht werden. Dabei waren auf deutscher Seite zunächst Erfolge zu verzeichnen, die Städte Nova Varoš und Pljevlja konnten von der 1. Gebirgs-Division und einem motorisierten Regiment „Brandenburger“ bereits am 5. und 6. Dezember eingenommen werden. Kurz darauf gelang auch die Erstürmung der Festung oberhalb von Prijepolje. ${ }^{23}$ An diesen Kämpfen nahmen bereits Freiwilligen-Bataillone lokaler Muslime teil, die laut Augenzeugenbericht aus Rache und Fanatismus darauf brannten, die Kommunisten umzubringen und auch die eingenommen Dörfer zu zerstören. ${ }^{24}$ Obwohl schließlich ein Kessel gebildet und die Stadt Višegrad erreicht werden konnte, waren viele Partisanen Richtung Norden entkommen - der gewünschte Erfolg blieb trotz der hohen Zahl von 2.926 „Feindtoten“ aus. ${ }^{25}$

Noch während des laufenden Unternehmens „Kugelblitz“ folgten Mitte Dezember 1943 das Unternehmen „Schneesturm“ und Anfang Januar 1944 „Waldrausch“. Alle drei Operationen erreichten ihr Ziel - die Zerschlagung der Partisanenverbände - trotz hoher Verluste der Gegner nicht. ${ }^{26}$ Das OKW ging in seinen Berichten von einer immensen Gesamtzahl von 30.000 gegnerischen Verlusten bei einer geringen Anzahl eigener Verluste (110 Gefallene, 201 Verwundete und 133 Vermisste) zwischen Dezember 1943 und Januar 1944 aus. $^{27}$ Dies deutet auf einen sehr ungleichen Kampf hin, der die Erschießung unschuldiger Zivilisten einschloss. Razzien sollten helfen, Partisanen und Partisaninnen aufzufinden

22 Vgl. Meyer, Blutiges Edelweiß, S. 558.

23 Vgl. ebd.

24 Dabei könnte es sich um die bereits in dieser Region (Sandžak) aufgestellten Freiwilligenlegionen der Waffen-SS gehandelt haben. Vgl. Meyer, Blutiges Edelweiß, S. 559.

25 Vgl. Meyer, Blutiges Edelweiß, S. 558.

26 Casagrande, Volksdeutsche SS-Division, S. 269.

27 Vgl. ebd., S. 269, 274. 
und an Straßensperren wurden Kontrollen durchgeführt. ${ }^{28}$ Allerdings wurde auch das I./SS-Freiwilligen-Gebirgsjäger-Regiment 13 am 6. Januar 1944 vollständig aufgerieben. ${ }^{29}$ Schmidhuber kommandierte ad interim, da Oberkamp aufgrund einer Erkrankung ausgefallen war, die Unternehmen „Kugelblitz“ und „Waldrausch“. Kumm ersetzte Oberkamp schließlich am 30. Januar 1944. ${ }^{30}$

\subsection{Unternehmen „Wegweiser“, 10. März 1944}

Das Unternehmen „Wegweiser“ markierte den Beginn der Einsätze der „Handschar“ in Südosteuropa. Ziel der Operation war es, die nördlich der Save vermutete Versorgungsbasis der Partisanen zu zerstören, da diese für ihre südlich der Save gelegenen Kräfte überlebenswichtig war. ${ }^{31}$ Dabei sollte das Gebiet um den Bošut von Partisanen „gesäubert“ werden. Diese wurden auf 2.000-2.500 Mann geschätzt. Angriffsbeginn war am 10. März um 4 Uhr, dabei wurde Jamena und Strošnici eingenommen, am zweiten Tag wurden die Angriffe auf die Partisanen fortgesetzt, um diese in das Dreick Save-Bošut-Studva (Flüsse) zu drängen. Als die Division bei Tagesanbruch des 13. März Bela Crkva erreichte, war das Dorf von den Partisanen bereits verlassen worden. Diese hatten aber alle Bewohner, da es sich mehrheitlich um „Volksdeutsche“ handelte, ermordet. Die Partisanen konnten schließlich der Umklammerung entkommen, das Bošut galt zwar vorerst als frei von Partisanen, es stand aber fest, dass diese bald zurückkehren würden. ${ }^{32}$

\subsection{Unternehmen „Save“, 14. März 1944}

Operation „Sava“ begann am 14. März 1943 mit der Überquerung der Save und markierte den Einzug der Division „Handschar“ in Bosnien. Dies war die wohl wichtigste Maßnahme unter dem Gesichtspunkt politischer Einflussnahme auf den Feind, die einen signifikanten Propagandaeffekt auf die Zivilbevölkerung und den Gegner haben sollte: Die Rückkehr muslimischer Freiwilliger in ihre

28 Vgl. Casagrande, Volksdeutsche SS-Division, S. 269f. Für Leibesvisitationen wurden „volksdeutsche“ Frauen, Rot-Kreuz-Schwestern sowie Dolmetscherinnen hinzugezogen. Vgl. ebd, S. 270.

29 Vgl. Michaelis, Gebirgs-Divisionen der Waffen-SS, S. 87.

30 Vgl. Casagrande, Volksdeutsche SS-Division, S. 271.

31 Sauberzweig, Divisionsbefehl Operation «Wegweiser», 29.2.1944, durch George Lepre zur Verfügung gestellt, S. 1, 7.

32 Vgl. Bernwald, Muslime, S. 185-187. 
Heimat und deren Befreiung vom „Bolschewismus“. Während dieser Zeit begingen Angehörige der Division „Handschar“ auch die meisten Kriegsverbrechen. ${ }^{33}$

\subsection{Unternehmen „Osterei“, 12. bis 20. April 1944}

Im Unternehmen „Osterei“ sollte die „Handschar“ zum letzten Mal mit jenen Teilen der Division im Einsatz stehen, die danach zur „Skanderbeg“ versetzt wurden. Bei dieser Anti-Partisanen-Aktion um Majevica - Priboj hatten das 27. und 28. Regiment zuerst schwere Verluste hinzunehmen. ${ }^{34}$ Bei der Lagebesprechung am 12. April in Mitrovići, im Nordosten Bosnien-Herzegowinas, trugen SS-Sturmbannführer Erich Braun und SS-Obersturmführer Walter Schaumüller den bosnischen Fez der Division „Handschar“, Schaumüller hingegen die albanische Filzkappe, die das albanische Bataillon und später die Soldaten der „Skanderbeg“ Division trugen. ${ }^{35}$ Das albanische Bataillon wurde während des laufenden Unternehmens am 17. April 1944 aus der Division herausgezogen. Am selben Tag konnte Srebrenik genommen werden und die Division bewegte sich Richtung Gračac und Bosna weiter. Am 20. April endete die Operation „Osterei““.36

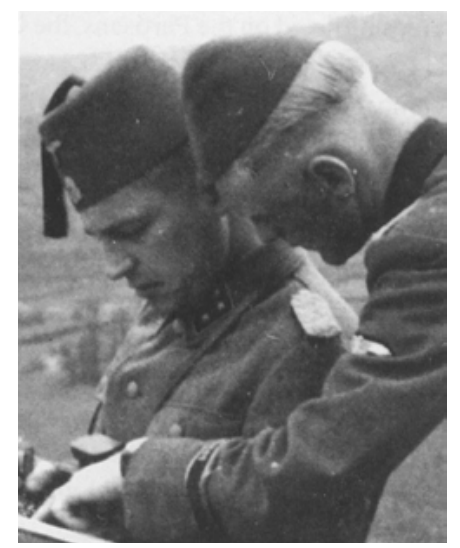

Abb. 46: SS-Sturmbannführer Erich Braun (links mit bosnischer Kopfbedeckung) und SS-Obersturmführer Walter Schaumüller (rechts mit albanischer Kopfbedeckung) während dem Unternehmen Osterei, in Mitrovići. ${ }^{37}$

33 Vgl. Lepre, Himmler's Bosnian Division, S. 151-162.

34 Vgl. Werhas, Mikulčić, „Handschar”, „Skanderbeg“, „Kama“, S. 15.

35 https://handzar.jimdo.com/drugi-svjetski-rat/ukratko-o-diviziji/erich-braun/ (Stand: 04.01.2019).

36 Vgl. Werhas, Mikulčić, „Handschar”, „Skanderbeg“, „Kama“, S. 15. Vgl. Lepre, Himmler’s Bosnian Division, S. 165-169.

37 https://handzar.jimdo.com/drugi-svjetski-rat/ukratko-o-diviziji/erich-braun/ (Stand: 04.01.2019). 


\subsection{Unternehmen Maibaum, 26. April bis 7. Mai 1944}

Kurz nach ihrer Rückkehr nach Bosnien stand die Division „Handschar“ im Unternehmen „Maibaum“, welches am 23. bzw. 26. April 1944 mit der „Prinz Eugen“ unter dem Kommando des V. SS-Gebirgskorps begann, im Einsatz. ${ }^{38}$ Dabei sollten die Divisionen, die zum ersten Mal in einer gemeinsamen Operation eingesetzt waren, gegen die von Tito in Ostbosnien konzentrierten Kräfte vorgehen. ${ }^{39}$ Im Raum Srebrenica-Vlasenica kam es zu schweren Kämpfen. ${ }^{40}$ Die Partisanenverbände waren den beiden Division zahlenmäßig überlegen und bereits durch die Engländer ausgerüstet. Dennoch mussten sie zahlreiche Verluste in ihren Reihen hinnehmen, 2.884 Tote, 1.434 Verwundete und 1.339 Gefangene. ${ }^{41}$

\subsection{Unternehmen „Rösselsprung“, 25. Mai bis zum 6. Juni 1944}

Das Unternehmen „Rösselsprung“ ist neben den Unternehmen „Schwarz“ und „Weiß“ das wohl bekannteste in Südosteuropa. Titos Hauptquartier bei Drvar sollte dabei ausgehoben und möglichst viele hochrangige Partisanenführer festgenommen bzw. getötet werden. Generaloberst Lothar Rendulić übertrug die Führung des Unternehmens General der Infanterie Ernst von Leyser. Im Zentrum der geplanten Einsätze stand das erste deutsche Luftlandemanöver im bosnischen Raum. ${ }^{42}$

Bei diesem Unternehmen spielte die „Prinz Eugen“ eine herausragende Rolle. Teilen der „Prinz Eugen“ gelang es, das Hauptquartier der Tito-Partisanen in Drvar zu zerstören sowie Versorgungseinrichtungen und „Waffen aller Art“ zu erbeuten. Es endete am 6. Juni 1944 zeitgleich mit der Landung der Alliierten in der Normandie. Für diesen Einsatz, in welchem sie unter Führung Rendulićs 6.240 Partisanen und Zivilisten töteten, wurden die Verbände der Wehrmacht und Waffen-SS vom Oberkommando der Wehrmacht gelobt. ${ }^{43}$ Denn, obwohl das Hauptziel, Tito zu

38 Vgl. Kaltenegger, Totenkopf und Edelweiss, S. 223. Vgl. Bernwald, Muslime, S. $191 \mathrm{f}$.

39 Vgl. Kaltenegger, Totenkopf und Edelweiß, S. 223.

40 Vgl. Kumm, Vorwärts Prinz Eugen, S. 173.

41 Vgl. Kaltenegger, Totenkopf und Edelweiß, S. $223 \mathrm{f}$.

42 Vgl. ebd., S. 225.

43 Vgl. Kumm, Vorwärts Prinz Eugen, 191f. Vgl. hierzu auch Karl Dieter Wolff, Das Unternehmen „Rößelsprung“. Der deutsche Angriff auf Titos Hauptquartier in Drvar im Mai 1944, Vierteljahrshefte für Zeitgeschichte, 4(1970), S. 476-509. 
töten oder zumindest gefangen $\mathrm{zu}$ nehmen, nicht erreicht wurde, werteten die Deutschen das Unternehmen „Rösselsprung“ als Erfolg. ${ }^{44}$

\subsection{Unternehmen „Vollmond“, 7. bis 12. Juni 1944}

Im Unternehmen „Vollmond“ ging es Sauberzweig darum, die kommunistischen Verbände an der Wiederherstellung ihrer Machtbasis im Gebiet um Posavina und Majevica zu hindern. Durch einen Angriff von Norden und Westen her sollten die Partisanen Richtung Drina abgedrängt werden. Dabei sollten sich I./27 und II./27 noröstlich von Pripoj sammeln und von dort Richtung Süden nach Bisoka und Sijenica vorgehen, I./28 ging auf den Höhen bei Priboj in Stellung. Allerdings war das dem I./27 und II./27 zugeteilte Gebiet zu weitläufig und die Partisanen konnten daher nicht aufgehalten werden und setzen ihren Vormarsch fort. ${ }^{45}$

\subsection{Unternehmen „Kornblume“, 14. Juni bis 6. August 1944}

Die Informationen zum Unternehmen „Kornblume“ sind spärlich und keine Berichte oder Verlustzahlen überliefert. Ziel war es, das Fruška Gora-Gebirge in Syrmien und den größten Teil des restlichen Syrmien nach Partisanen zu „durchkämmen“, und diese in jenem Gebiet operierenden mobilen Partisanenformationen zu zerschlagen. ${ }^{46}$

\subsection{Unternehmen „Rübezahl“/,Hackfleisch“, vorgesehener Beginn 7. /24. Juli, wirklicher Beginn: 4.8. bis 31.8.1944}

Auch das Unternehmen „Rübezahl“ wurde im albanisch-montenegrinischbosnischen Grenzgebiet durchgeführt und stand unter dem Zeichen der am 7. Juli 1944 im Stab des Oberbefehlshabers Südost geäußerten Absicht einer „Generalbereinigung in Montenegro“. ${ }^{47}$ Dabei wurden die im bosnischen Teil

44 Vgl. Kumm, Vorwärts Prinz Eugen, S. 194. Vgl. hierzu auch Erfahrungsbericht „Rösselsprung“, Kaltenegger, Totenkopf und Edelweiß. S. $240 \mathrm{f}$.

45 Vgl. Bernwald, Muslime, S. 195f. Vgl. hierzu auch Divisionsbefehl „Vollmond“, 13. SSDivision, nach: Kaltenegger, Totenkopf und Edelweiss, S. 237.

46 Vgl. http://www.lexikon-der-wehrmacht.de/Gliederungen/GebirgsdivisionenSS/13SSGebD. htm (Stand: 17.1.2018).

47 Vgl. Schmider, Partisanenkrieg, S. 504 f. 
durchgeführten Kampfhandlungen des Unternehmens „Rübezahl“ als „Hackfleisch“ bezeichnet. Wegen der 1. Gebirgs-Division, die in einem noch laufenden Operationszyklus in Griechenland eingesetzt war und somit nicht verlegt werden konnte, wurde der Beginn des Unternehmens auf den 24. Juli 1944 verschoben. ${ }^{48}$ Es kam zu weiteren Verzögerungen, und das Unternehmen „Rübezahl“ lief schließlich erst Anfang August 1944 „bei zum Teil hartnäckigem Feindwiderstand" an und sollte am 26. August abgeschlossen sein. ${ }^{49}$ In diesem Unternehmen kämpften Einheiten der Divisionen „Prinz Eugen“, „Skanderbeg“ und anfänglich auch der „Handschar“ gemeinsam mit Teilen 1. Gebirgs-Division, des 2. Regiments „Brandenburg“ und Teilen der 181. Infanterie-Division im montenegrinischen Teil des Sandžak und bosnischen Gebieten mit dem Ziel, die Partisanen an einem Einfall in serbisches Territorium zu hindern. ${ }^{50}$ Die Kämpfe zogen sich bis in die Grenzgebiete zu „Großalbanien“, wo die 2., 5. und 17. Tito-Division in Südserbien zwischen Mitrovica und Raška nach Osten über den Ibar vorstießen, wobei das SS-Gebirgs-Jäger-Regiment 14 und Teile der 1. Gebirgs-Division „in harte Kämpfe verwickelt" wurden. ${ }^{51}$ Auch hier wurde die enorme Überlegenheit des Gegners mit drei Verbänden Titos und fünf „altserbischen“ Divisionen offensichtlich. Die deutschen Einheiten sahen sich nach Schmider nun einer regelrechten Armee gegenüber. ${ }^{52}$

Das Unternehmen betraf auch den „neualbanischen“ Raum, wo das I./Waffen-Geb.Jäg.Rgt. der SS 51 gegen eine Bande von 300 Mann im Raum Tropoje, 22 Kilometer westlich von Gjakova/Đjakovica eingesetzt wurde. ${ }^{53}$ Nach einem Teilerfolg hatte die „Skanderbeg“ die Aufgabe, im „gesäuberten“ Raum die Straße Prizren-Pejë/Peć-Andrijevica zu sichern. Die Rückeroberung der Straße von Pejë/Peć nach Podgorica hingegen konnte nicht mehr umgesetzt werden. ${ }^{54}$ Das Fazit des Unternehmens war für die Deutschen ernüchternd: Weder die Einkesselung noch

48 Vgl. ebd., S. 505.

49 Vgl. Tagesmeldung Gen. Kdo. XXI. Geb.A.K. an Pz.AOK2, 14.8.44, NARA, T314/664, Bl. 533. Vgl. Schmider, Partisanenkrieg, S. 508.

50 Fernschreiben an SS-Div. „Skanderbeg“, undatiert (entweder 28. oder 29.8.1944), NARA, T314/664, F. 643. Vgl. Meyer, Blutiges Edelweiß, S. 647. Vgl. Lepre, Himmler’s Bosnian Division, S. 241. Vgl. Kumm, Vorwärts Prinz Eugen, S. 241.

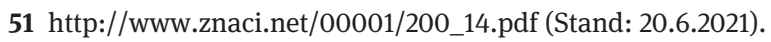

52 Vgl. Schmider, Partisanenkrieg, S. 512.

53 Vgl. Tagesmeldung Gen. Kdo. XXI. Geb.A.K. an Pz.AOK2, 14.8.44, NARA, T314/664, Bl. 533.

54 Vgl. Fernschreiben Gen.Kdo. XXI. Geb.A.K. an SS-Div. „Skanderbeg“, undatiert (28. oder 29.8.1944), NARA, T314/664, Bl. 643. 
die Vernichtung der gegnerischen Verbände waren gelungen und in ihrem Marsch Richtung Serbien waren sie nur für wenige Tage aufgehalten worden. ${ }^{55}$

\subsection{Unternehmen „Draufgänger“, 18. bis 28. Juli 1944}

Ziel des Unternehmens „Draufgänger“ vom 18. bis 28. Juli 1944 war die Zerstörung der Nachschubbasis der kommunistischen Partisanen in Berane im nordöstlichen Montenegro, um Tito im weiteren Kriegsverlauf nicht die Initiative zu überlassen. ${ }^{56}$ An diesem Unternehmen beteiligten sich Einheiten verschiedener Verbände, insbesondere aber die frisch aufgestellte Division „Skanderbeg“, die nach Schmider, „an dieser Aufgabe jedoch in spektakulärer Weise scheitern“ sollte. ${ }^{57}$ Neben der „Skanderbeg“ waren auch Teile der 1. Gebirgs-Division und der „Prinz Eugen“ bei dieser Operation eingesetzt. ${ }^{58}$ Den Oberbefehl über das Unternehmen hatte Phleps. ${ }^{59}$

Der Angriff auf Berane mit anschließender Einnahme wurde letztlich für den 23. Juli 1944 festgesetzt. ${ }^{60}$ Die „Skanderbeg“ hatte unter anderem den Auftrag, die erreichte Linie Praninica-Srit-Visibaba-Lukin zu halten, um so bereits eine günstige Ausgangsstellung für das Unternehmen „Rübezahl“ zu schaffen. Mit einer Zangenbewegung hätten in diesem Unternehmen zwei PartisanenDivisionen einerseits von Kacuber-Petnjica und andererseits von Andrijevica über Lubnice gegen Berane eingeschlossen und vernichtet werden sollen. ${ }^{61}$

Bei diesem Unternehmen wurde aber nun offensichtlich, was sich bereits im Juni 1944 abgezeichnet hatte: Die Kräfteverhältnisse hatten sich endgültig zugunsten der Partisanen verschoben. In seinem Gefechtsbericht lobte Schmidhuber den Feind als eine ,einheitlich englisch uniformierte und sehr gute ausgerüstete und ausgebildete Truppe unter auffallend guter Führung“. Weiter schrieb er: „Der Ausbildungsstand und der Gefechtswert dieser militarisierten

55 Schmider schreibt von einigen Tagen bis gut einer Woche Verzögerung. Vgl. Schmider, Partisanenkrieg, S. 510. Vgl. auch Lepre, Himmler's Bosnian Division, S. 242-247.

56 Vgl. Schmider, Partisanenkrieg, S. 505.

57 Ebd.

58 Vgl. Kriegsgefangenenbericht II Schmidhuber, 19.8.1945, VA HEM.OK.BOJCKA, 72/1/2/3.

59 Vgl. Kriegsgefangenenbericht Graf, VA, HEM.OK.BOJCKA, 72/2-1/32. Vgl. zum Unternehmen „Draufgänger“ ausführlich Frolov, Pod barjakom Skenderbega, S. 153-156.

60 Vgl. Meldekopf Rožaje an Hptm. Bendl, 21./22.7.1944, BA-MA, RS 3-21/2, S. 24-26.

61 Vgl. Gefechtsbericht für das Unternehmen „Draufgänger“ vom 18.7.-28.7.1944, Schmidhuber, Lagebeurteilung 22.7.44, NARA, T314/664, F. 283. 
Banditen hat sich als überraschend gut erwiesen. Truppe und Führung müssen einer vollwertigen europäischen Kampftruppe gleichgestellt werden. “62 Seiner Ansicht nach zählten die Kämpfe im Unternehmen „Draufgänger“ zu den bisher härtesten und wären nicht vergleichbar mit solchen wie beim Unternehmen „Rösselsprung“. ${ }^{63}$ Insbesondere Führer, Unterführer und Mannschaften des SS-Freiw.Geb.Jäg.Rgt. 14, das Pz.Gren.Sturm-Btl. 2, die aus deutscher Kriegsgefangenschaft entlassenen Albaner und Teile des Waffen-Geb-Jäg.Rgt. der SS 51 „Skanderbeg“, dessen Angehörige bereits in der „Handschar“ gedient hatten, hätten sich im Kampf ausgezeichnet gehalten. ${ }^{64}$

Das Massaker, das während des Unternehmens „Draufgänger“ am 28. Juli 1944 an der montenegrinischen Bevölkerung des Dorfes Velika, unter anderem von Einheiten der „Skanderbeg“, etwa von Teilen des Waffen-Geb.Jäg.Rgt. der SS 51, ${ }^{65}$ verübt wurde, rechtfertigte Schmidhuber damit, die lokale Bevölkerung hätte die Partisanen unterstützt. ${ }^{66}$

Das Generalkommando des XXI. Gebirgskorps zog bereits am 13. August eine negative Bilanz: „Dem Unternehmen ,Draufgänger` blieb ein Erfolg nach anfängliche[m], günstige[m] Anlaufen versagt. Es trat ein ernster Rückschlag ein. “67 Die hohe Zahl von 400 Desertionen in diesem Unternehmen kann vor diesem Hintergrund als Vorbote für die erst noch einsetzenden Massendesertionen innerhalb der „Skanderbeg“ im Folgemonat gewertet werden. ${ }^{68}$

62 Ebd. Schmidhubers Aussage lässt Zweifel aufkommen, ob hier überhaupt noch von „Partisanen“ gesprochen werden kann. Peter Lieb gibt zu dieser Problematik in seiner Publikation „Konventioneller Krieg oder NS- Weltanschauungskrieg?“ einen guten Überblick. Vgl. Lieb, Peter, Konventioneller Krieg oder NS- Weltanschauungskrieg?.

63 Gefechtsbericht für das Unternehmen „Draufgänger“ vom 18.7.-28.7.1944, Schmidhuber, Lagebeurteilung 22.7.44, NARA, T314/664, F. 283.

64 Abschließender Gefechtsbericht des Unternehmens „Draufgänger“ vom 18.-28. Juli 1944, Schmidhuber, NARA, T314/664, F. $293 \mathrm{f}$.

65 Nach Gordana Kostic und Laurent Latruwe befanden sich in diesem Regiment besonders viele Albaner, die aus der nächsten Umgebung, aus Plav, Gusinje sowie Peć/Peja, stammten. Vgl. Kostic, Latruwe, Division Skanderbeg, S. 175.

66 Vgl. Gefechtsbericht für das Unternehmen „Draufgänger“ vom 18.7.-28.7.1944, Schmidhuber, Lagebeurteilung 22.7.44, NARA, T314/664, F. 283.

67 Lagebericht Gen.Kdo. XXI. Geb.A.K. an Pz.AOK 2, 13.8.44, NARA, T314/664, Bl. 514. 68 Schmider, Partisanenkrieg, S. 506. 


\subsection{Unternehmen „Fuchsjagd“, 17. August 1944}

Bei „Fuchsjagd“ handelt es sich um ein kleineres Unternehmen, dessen Beginn auf den 17. August 1944 angesetzt war. An ihm lässt sich gut nachvollziehen, wie kleinräumig der Partisanenkrieg geworden war. Auch hatte es nicht mehr die Zerschlagung der Partisaneneinheiten zum Ziel, man beschränkte sich auf deutscher Seite darauf, durch „Störaktionen“ dem Feind Verluste zuzufügen und ihn an der „ungestörten Organisation seiner Verbände“ $z u$ hindern. ${ }^{69}$ Die unter britischer Führung stehenden und mit automatischen Waffen ausgerüsteten Partisanen waren Ende August 1944 der Division „Skanderbeg“ weit überlegen. ${ }^{70}$ Hauptziel dieses Unternehmens war es daher nicht mehr, die Partisanen zu vernichten, sondern wenn möglich an Ausrüstungsdepots des Gegners zu gelangen, um die eigene prekäre Versorgungssituation zu entschärfen. ${ }^{71}$ In diesem Unternehmen der „Skanderbeg“ erreichte die Kampfgruppe „Nord“ ohne Feindberührung Vlashaj, die Kampfgruppe „West“ wehrte schwache Feindvorstöße gegen Perlati ab. Die Kampfgruppe „Süd“ musste ihre Position wegen starker Partisanenangriffe aufgeben und auf die Sperrstellung beiderseits Maqellarë zurückgehen. Durch die starken Angriffe versagten auch die den deutschen Truppen angegliederten „nationalen Banden“. Im Osten gelang es den Partisanen, die Kampfgruppe „Ost“ zurückzudrängen und mehrere Höhen im Zuge der Sperrstellung zu nehmen. Die drei in diesem Abschnitt eingesetzten albanischen SS-Kompanien hielten den feindlichen Angriffen nicht stand, waren schließlich überall versprengt und hatten 300 Vermisste $\mathrm{zu}$ beklagen. ${ }^{72}$

69 „Betr.: Unternehmen ,Fuchsjagd““, Gen.Kdo. XXI. Geb.A.K., 16.8.44, NARA, T314/664, F. 544.

70 Vgl. ebd., F. 544.

71 Ebd., F. 548. Zur Aufstellung der wiederum auf vier Gruppen verteilten Kräfte vgl. ebd., F. 545.

72 Hauptmann Hausen, Gen.Kdo. XXI. Geb.A.K., 22.8.1944, Kriegstagebuch Nr. 12, BA 59 824/2. 


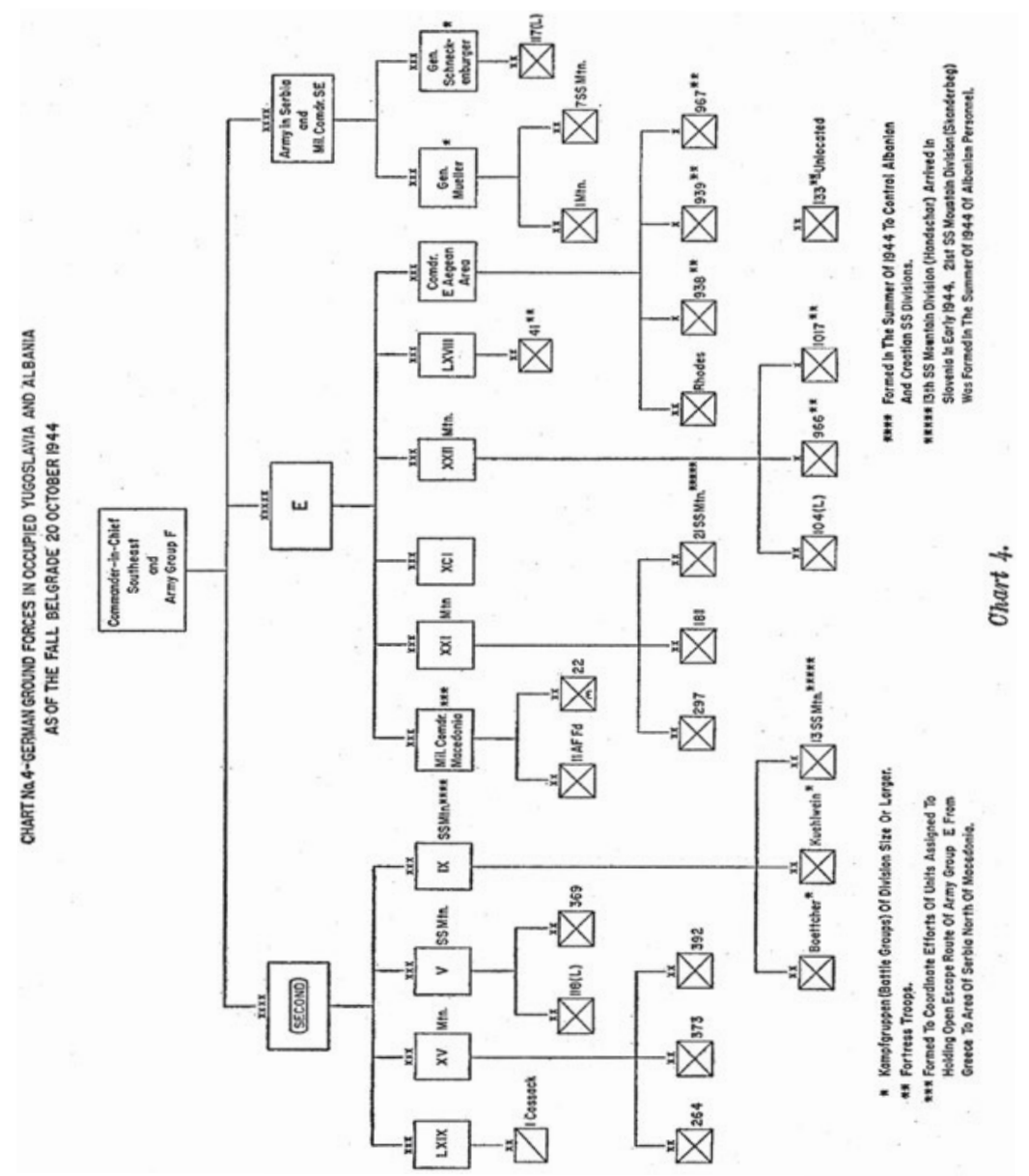

Abb. 47: Gesamtüberblick deutscher Bodentruppen in Südosteuropa, Oktober $1944 .^{73}$

73 Robert M. Kennedy, German Antiguerrilla Operations in the Balkans, Department of the Army Pamphlet NO. 20-243, Washington D.C.: Department of the Army 1954, S. 68. 